\title{
A perspective on femoroacetabular impingement
}

\author{
Thomas C. B. Pollard
}

Published online: 15 March 2011

(C) ISS 2011

\begin{abstract}
Although Smith-Petersen described impingement of the native hip in 1936 [1], the success of replacement arthroplasty of the hip dominated the orthopaedic literature until the early contemporary reports of femoroacetabular impingement (FAI) at the turn of the millennium [2]. Subsequently, an exponential increase in interest in the subject has occurred, as demonstrated by the annual number of Pubmed hits for FAI, which reached 168 in 2010 alone. This interest is perhaps not surprising when one considers that FAI represents a new diagnosis for young adults with hip pain, an opportunity for advanced imaging and surgical techniques, and perhaps most tantalisingly, the possibility of altering the natural history of degenerative joint disease and limiting progression to endstage osteoarthritis (OA). With the explosion of recent interest in FAI, there are many controversies and unresolved issues that will be the targets for research over the next decade.
\end{abstract}

\section{Aetiology of FAI}

Femoroacetabular impingement may occur secondary to paediatric hip disease or trauma, but the vast majority of cases have no underlying known cause and may be considered "primary FAI". A hip with symptomatic FAI is likely to differ from a "normal" hip through a combination of its morphology, durability of its articular cartilage and labrum, and demands placed upon it, with regard to the

T. C. B. Pollard $(\bowtie)$

Nuffield Department of Orthopaedics, Rheumatology,

and Musculoskeletal Sciences, Nuffield Orthopaedic Centre,

University of Oxford,

Oxford OX3 7LD, UK

e-mail: Tom.Pollard@ndorms.ox.ac.uk range of movement and activity level of the patient. Recent evidence suggests a strong genetic component to FAI [3], which is in keeping with the known genetic predisposition to hip OA. Whether abnormal morphology is determined at conception, or is acquired during skeletal development, is not known. It is conceivable that there is a genetic predisposition to subclinical slipped capital femoral epiphysis, for example. However, not all hips with abnormal joint morphology develop symptoms. A cam deformity is a common finding (approximately 20\% incidence) in asymptomatic male subjects [4-7] and patients with FAI often have similar deformities in the contralateral asymptomatic hip [8]. This supports the notion that additional variables, such as the vulnerability of the labrum and articular cartilage to injury, and activity level, are important in modulating whether abnormal morphology results in symptoms. The importance of activity type and intensity needs further investigation. It stands to reason that an activity such as hurdling will potentially damage an at-risk hip by increasing the frequency and severity of impingement episodes. Better ways of grading activity are required in order to classify individuals. With regard to morphology, very little is known regarding the development of the nondysplastic hip through childhood and adolescence, and prospective studies during skeletal development may help to determine whether morphology is dependent on activity or other factors. Longitudinal studies of well-characterised cohorts will improve our understanding of how FAI develops.

\section{Difficulties with imaging}

It is crucial to appreciate that making a diagnosis of FAI relies on a consensus of clinical findings and imaging 
abnormalities. Given the prevalence of "abnormality" in the asymptomatic population [7], in common with the spine, MRI will show abnormalities that are not necessarily clinically important. It is important not to base management decisions on imaging findings alone.

Particular problems with imaging relate to the diagnosis of abnormal morphology, the sensitivity and specificity for labral tears, and the detection of acetabular cartilage damage. Consensus is needed on how best to image morphology, as there is inherent variability in all methods and the thresholds of what is normal, borderline, and abnormal morphology are not clear. Imaging protocols vary between units, in terms of both modality employed and technique within that modality. Parameters measured are sensitive to the imaging modality, patient positioning and observer reliability. Consequently, this is translated as confusing literature. The alpha angle, for example, was originally described from an MRI study, with a non-genderspecific cut-off of $50^{\circ}$ suggested [9]. However, the reliability of its measurement has been questioned [10, 11]. The $50^{\circ}$ threshold may be extrapolated to lateral radiographs [12], but this assumption has not been validated and the abnormal threshold may be as high as $62^{\circ}$ with this method [5]. A threshold of $55^{\circ}$ has been suggested based on CT [13]. The situation is just as confused when one considers the acetabulum, because of the sensitivity to pelvic positioning and tilt. It is unclear how well a cross-over sign on an AP pelvic radiograph correlates with acetabular retroversion assessed by CT [14]. Does it matter whether the patient is standing or supine when imaged and should that individual's pelvic tilt be standardised to comply with perceived normality, thereby affecting the projection of their acetabulum, and the interpretation of the acetabular version?

With regard to the acetabular labrum, there has been much interest in the radiology literature concerning the optimal method of detection of labral pathology. Normal labral sulci may be reported as tears, leading to poor specificity. In general, most labral tears will have associated abnormalities and it is relatively rare to have a tear in isolation. From a surgical viewpoint, evidence of associated abnormalities, such as abnormal morphology, herniation pits [15] and labral ossification, together with a positive response to an intra-articular local anaesthetic injection, is usually enough to proceed with surgery. Pre-operative identification of a large labral detachment is useful as repair should be considered.

The limited literature as regards outcomes following treatment for FAI indicates that the most important predictor is the degree of pre-existing cartilage damage. In FAI, this poses a problem as delaminating acetabular cartilage (carpet lesion) is hard to detect with MRA unless the flap is detached, allowing contrast material underneath it. Sensitivity for delamination assessed by MRA is only $22 \%$ [16]. Potentially more sophisticated MR techniques, such as dGEMRIC [17-20], T1rho and T2 mapping, will enable more accurate identification of cartilage damage preoperatively. A number of studies have investigated the role of dGEMRIC in FAI. In asymptomatic hips with and without cam deformities, but with no evidence of OA, the dGEMRIC index was lower in cam hips in the anterosuperior acetabular cartilage and appeared to correlate with the alpha angle measured from lateral radiographs [17]. Furthermore, hips with a positive impingement sign had a lower total joint dGEMRIC index [17]. In symptomatic hips with FAI, the dGEMRIC index correlated with pain and alpha angle [19]. However, in an association study of dGEMRIC with intra-operative findings, localized dGEMRIC values were only mildly associated with specific regions of macroscopic damage [20]. Therefore, the interpretation of dGEMRIC maps, and the technique's role as an imaging biomarker of early OA, needs further evaluation, but does offer great promise.

\section{Treatment of FAI: short-term outcomes}

Non-operative management of FAI includes rest, antiinflammatory medication and core muscle strengthening. Patients with persisting symptoms are candidates for surgery, although there has been no comparative study of non-operative and operative management. Surgery for FAI aims to treat the secondary damage to the labrum and articular cartilage, and address the underlying joint deformity. The labrum may be debrided or repaired. Unstable articular cartilage may undergo debridement, or stabilisation with thermal chondroplasty or fibrin glue. For fullthickness lesions, microfracture is the mainstay, but some centres are attempting resurfacing using matrix-based techniques. Pincer deformities may be treated by acetabular rim-trimming, and cam deformities of the femoral neck by osteochondroplasty. These procedures are all possible through a variety of surgical approaches that include open dislocation, anterior arthrotomy, and arthroscopy. Reverse periacetabular osteotomy, rather than rim-trimming, may be appropriate if there is acetabular retroversion. The evidence for FAI surgery is largely based on case series with no controlled trials, with only 23 articles (all retrospective; 1 level II, 2 level III, 20 level IV) suitable for inclusion in the systematic review by $\mathrm{Ng}$ et al. [21]. Major complications are rare. Both open and arthroscopic treatments were found to improve function and scores in all studies, although patient dissatisfaction rates and patients with no improvement of symptoms ranged from 0 to $31 \%$. Progression of OA ranges from 0 to $33 \%$ and conversion rates to total hip replacement occur in up to $30 \%$ of cases. Factors associated 
with a good outcome include minimal radiographic OA, limited cartilage damage at surgery, young age and labral repair (rather than debridement). Whilst advanced chondral damage is associated with poor outcome, not all patients with such damage do badly. As for the knee, hip arthroscopy with appropriate pre-operative counselling may be a reasonable option for the young patient with early to moderate OA secondary to FAI who is not prepared to contemplate replacement surgery. However, it is clear that for the non-arthritic case, more accurate pre-operative characterisation of cartilage damage is likely to improve case selection and surgical outcomes. Thus, although the surgical management of FAI appears to be safe and effective, the quality of evidence is relatively poor. In government-funded healthcare systems such as the UK, FAI surgery may come under scrutiny as cost-savings are sought. Robust data regarding clinical effectiveness are urgently required.

With regard to the detailed surgical strategies in the treatment of FAI, the literature reports a variety of approaches, and there is inconsistency in the method of recording of pre-operative and operative data and clinical outcome measures. This limits comparison between studies. There is no uniform method of describing labral and cartilage lesions with respect to severity, size and location. Thus, the current literature does not provide answers to key questions, such as whether treatment should be performed open or arthroscopically, whether the labrum should be repaired when possible or resected in all cases, and whether correction of bony abnormality is mandatory or whether isolated management of the presumed consequences (i.e. damage to the labrum and acetabular cartilage) is all that is required. There is no consensus on what to do with a carpet lesion and how to treat severe acetabular cartilage lesions. Whether it is necessary to address pincer and cam deformities in the same hip, or just address one side of the joint, has also not been investigated. These questions will remain unanswered until controlled studies are performed, and realistically a multicentre trial will be required.

\section{Treatment of FAI: prevention of OA?}

Specific abnormalities associated with FAI are recognised as biomechanical risk factors for the development of OA of the hip. Prospective data to support these historical observations is now becoming evident through longitudinal cohort studies $[22,23]$. Given the anticipated increasing burden of hip OA, the possibility that the treatment of FAI may reduce progression to end-stage $\mathrm{OA}$ is exciting. Nevertheless, although there is acceptance that FAI does predispose to OA, it remains unclear as to whether intervention will alter natural history and there is no good-quality prospective evidence to answer this question either way. One cannot yet advise patients that such surgery will prevent progression and save them from arthroplasty.

The difficulty in answering this issue relates to the assessment of progression. A reliable method of assessing disease progression in the short term is necessary. Radiographic studies are limited because they require long-term follow-up in large numbers of patients. From a practical perspective, a clinical trial would need a rapid assessment of efficacy to yield a result. There is an urgent need for validation of alternative markers of progression for introduction into trials. Newer MRI techniques as outlined above may potentially play roles as biomarkers of early OA. These techniques may help case selection, and act as surrogate markers of outcome and disease progression.

In order to stratify cases appropriately in clinical trials, reliable classification of morphology, activity and preexisting cartilage damage, using validated and functional definitions, is required. Accurate classification of these variables in order to phenotype an individual, combined with observation of progression, will enable us to work out what the most important predictors of progression are and therefore which should be modified.

\section{Conclusion}

In summary, FAI is an established cause of hip pain and degenerative hip disease. Surgical outcomes appear to be generally good, but there is a marked paucity of comparative literature. In order to refine surgical strategies and improve outcomes, pre-operative imaging must be improved to stage patients accurately and classify their morphology, and assessment of intra-operative findings and post-operative outcomes must be standardised. Only through recruitment of such well-characterised cohorts into clinical trials will it be possible to address the current uncertainties in the management of FAI.

\section{References}

1. Smith-Petersen MN. Treatment of malum coxae senilis, old slipped upper femoral epiphysis, intrapelvic protrusion of the acetabulum, and coxa plana by means of acetabuloplasty. J Bone Joint Surg Am. 1936;18:869-80.

2. Ito K, Minka 2nd MA, Leunig M, Werlen S, Ganz R. Femoroacetabular impingement and the cam-effect. A MRIbased quantitative anatomical study of the femoral head-neck offset. J Bone Joint Surg Br. 2001;83:171-6.

3. Pollard TCB, Villar RN, Norton MR, Fern ED, Williams MR, Murray DW, et al. Genetic influences in the aetiology of femoroacetabular impingement: a sibling study. J Bone Joint Surg Br. 2010;92:209-16. 
4. Gosvig KK, Jacobsen S, Sonne-Holm S, Gebuhr P. The prevalence of cam-type deformity of the hip joint: a survey of 4151 subjects of the Copenhagen Osteoarthritis Study. Acta Radiol. 2008;49:436-41.

5. Pollard TCB, Villar RN, Norton MR, Fern ED, Williams MR, Simpson DJ, et al. Femoroacetabular impingement and classification of the cam deformity: the reference interval in normal hips. Acta Orthop. 2010;81:134-41.

6. Hack K, Di Primio G, Rakhra K, Beaule PE. Prevalence of camtype femoroacetabular impingement morphology in asymptomatic volunteers. J Bone Joint Surg Am. 2010;92:2436-44.

7. Kang AC, Gooding AJ, Coates MH, Goh TD, Armour P, Rietveld J. Computed tomography assessment of hip joints in asymptomatic individuals in relation to femoroacetabular impingement. Am J Sports Med. 2010;38:1160-5.

8. Allen D, Beaule PE, Ramadan O, Doucette S. Prevalence of associated deformities and hip pain in patients with cam-type femoroacetabular impingement. J Bone Joint Surg Br. 2009;91:589-94.

9. Notzli HP, Wyss TF, Stoecklin CH, Schmid MR, Treiber K, Hodler J. The contour of the femoral head-neck junction as a predictor for the risk of anterior impingement. J Bone Joint Surg Br. 2002;84:556-60.

10. Lohan DG, Seeger LL, Motamedi K, Hame S, Sayre J. Cam-type femoral-acetabular impingement: is the alpha angle the best MR arthrography has to offer? Skeletal Radiol. 2009;38:855-62.

11. Audenaert EA, Baelde N, Huysse W, Vigneron L, Pattyn C. Development of a three-dimensional detection method of cam deformities in femoroacetabular impingement. Skeletal Radiol 2010;doi:10.1007/s00256-010-1021-2.

12. Clohisy JC, Carlisle JC, Beaule PE, Kim YJ, Trousdale RT, Sierra $\mathrm{RJ}$, et al. A systematic approach to the plain radiographic evaluation of the young adult hip. J Bone Joint Surg Am. 2008;90 (Suppl 4):47-66.

13. Beaule PE, Zaragoza E, Motamedi K, Copelan N, Dorey FJ. Three-dimensional computed tomography of the hip in the assessment of femoroacetabular impingement. J Orthop Res. 2005;23:1286-92.

14. Werner CM, Copeland CE, Stromberg J, Ruckstuhl T. Correlation of the cross-over ratio of the cross-over sign on conventional pelvic radiographs with computed tomography retroversion measurements. Skeletal Radiol. 2010;39:655-60.

15. Panzer S, Esch U, Abdulazim AN, Augat P. Herniation pits and cystic-appearing lesions at the anterior femoral neck: an anatomical study by MSCT and microCT. Skeletal Radiol. 2010;39:64554.

16. Anderson LA, Peters CL, Park BB, Stoddard GJ, Erickson JA, Crim JR. Acetabular cartilage delamination in femoroacetabular impingement. Risk factors and magnetic resonance imaging diagnosis. J Bone Joint Surg Am. 2009;91:305-13.

17. Pollard TCB, McNally EG, Wilson DC, Wilson DR, Madler B, Watson $\mathrm{M}$, et al. Localized cartilage assessment with threedimensional dGEMRIC in asymptomatic hips with normal morphology and cam deformity. J Bone Joint Surg Am. 2010;92:2557-69.

18. Bittersohl B, Steppacher S, Haamberg T, Kim YJ, Werlen S, Beck M, et al. Cartilage damage in femoroacetabular impingement (FAI): preliminary results on comparison of standard diagnostic vs delayed gadolinium-enhanced magnetic resonance imaging of cartilage (dGEMRIC). Osteoarthritis Cartilage. 2009;17:1297-306.

19. Jessel RH, Zilkens C, Tiderius C, Dudda M, Mamisch TC, Kim YJ. Assessment of osteoarthritis in hips with femoroacetabular impingement using delayed gadolinium enhanced MRI of cartilage. J Magn Reson Imaging. 2009;30:1110-5.

20. Bittersohl B, Hosalkar HS, Apprich S, Werlen SA, Siebenrock KA, Mamisch TC. Comparison of pre-operative dGEMRIC imaging with intra-operative findings in femoroacetabular impingement: preliminary findings. Skeletal Radiol;doi:10.1007/ s00256-010-1038-6.

21. Ng VY, Arora N, Best TM, Pan X, Ellis TJ. Efficacy of surgery for femoroacetabular impingement: a systematic review. Am J Sports Med. 2010;38:2337-45.

22. Bardakos NV, Villar RN. Predictors of progression of osteoarthritis in femoroacetabular impingement: a radiological study with a minimum of ten years follow-up. J Bone Joint Surg Br. 2009;91:162-9.

23. Nicholls AS, Kiran A, Pollard TCB, Hart D, Arden CPA, Spector $\mathrm{T}$, et al. The association between hip morphology parameters and 19-year risk of end-stage osteoarthritis in the hip: a nested casecontrol study. J Bone Joint Surg Br Proceedings 2011; in press. 